\title{
Editorials
}

\section{Cardiopulmonary resuscitation in primary and community care during the COVID-19 pandemic}

Severe acute respiratory syndrome coronavirus 2 (SARS-CoV-2), the virus that causes coronavirus disease 2019(COVID-19), can be spread by droplets or aerosols, particularly through direct or close contact and aerosol generating procedures (AGPs). Supplies of personal protective equipment $(\mathrm{PPE})^{2}$ are limited, raising uncertainties in clinical judgement about the balance between benefit (to the patient) and risk (to the healthcare worker) during medical procedures, such as cardiopulmonary resuscitation (CPR) undertaken without adequate protection during the COVID-19 pandemic. Lack of PPE has caused intense anxiety in view of the increased number of deaths in healthcare workers including in primary and community care. ${ }^{2}$

CPR can be a complex intervention comprising airway management, ventilation, chest compressions, drug therapy, and defibrillation. ${ }^{3}$ While the intubation component of CPR is almost universally classified as an AGP, there is controversy around the risk of chest compression (to the person performing it, and to other staff and bystanders). ${ }^{4}$

Risks to healthcare workers will vary depending on the setting where such individuals work (primary or community care versus hospital-based care); and whether the individual works in an environment where AGPs are performed. The key concern for healthcare professionals is based on the possibility of aerosol generation with chest compressions and the risks associated with close physical contact with the patient. In addition, others who aid in the CPR effort, such as in primary or community care settings, even if not performing chest compressions themselves, may be in close physical proximity so as to be exposed as bystanders. Confusion has arisen because of varied guidance on CPR in different regions. For example, healthcare staff in one UK region were told not to start chest compressions or ventilation in patients having a cardiac arrest if they had either confirmed or suspected COVID-19 unless

"The key concern for healthcare professionals is based on the possibility of aerosol generation with chest compressions and the risks associated with close physical contact with the patient.

staff were wearing full (that is, aerosol and droplet protective) PPE, including a respirator mask (FFP3 mask), full gown with long sleeves, gloves, and eye protection. ${ }^{5}$

Public Health England (PHE) have issued various guidance documents on PPE since January 2020.6 Initial guidance suggested that chest compressions and defibrillation were not AGPs and could be carried out without full PPE. New guidance published on 24 April again designated chest compressions as a nonAGP, but recommended that healthcare organisations may choose to advise their clinical staff to wear FFP3 respirators, gowns, eye protection, and gloves when performing chest compressions. However, the same guidance also strongly advised that there should not be potential delays in delivering this 'life-saving intervention'.

The International Liaison Committee on Resuscitation (ILCOR) recently published a systematic review that aimed to identify the risk of potential transmission from chest compression, defibrillation, and CPR. ${ }^{4}$ Of the 11 studies included in that review, five were case reports (a very weak design) describing a total of nine healthcare workers who developed a serious respiratory disease after performing CPR on a patient with that disease. Three were simulation studies on manikins. Of the other three two were retrospective cohort studies and one was a case-control study. The review rightly concludes, the quality of primary evidence was thus low or very low, and studies provided only indirect evidence with none directly reporting on transmission of SARS-CoV-2. The review recommended that absence of evidence should not be interpreted as providing evidence that chest compressions are non-aerosol generating. ${ }^{4}$

The PHE recommendation that chest compression is not an AGP is thus not evidence based. The recommendations in the PHE guidance also conflict with the majority of guidelines and position statements we have reviewed, which classify CPR as an AGP or possible AGP (systematic review in preparation; personal communication, S Straube, 2020). These include the US Centers for Disease Control and Prevention, the European Society of Intensive Care Medicine, and the Society of Critical Care Medicine. ${ }^{7,8}$ The latest World Health Organization (WHO) COVID-19 specific guidance classifies CPR as an AGP.1 Resuscitation Council UK has also recommended full PPE including FFP3 mask, full gown with long sleeves, gloves, and eye protection, as well as shoe protection for chest compressions. ${ }^{9}$ They have based their guidance on the $\mathrm{WHO}$ and the ILCOR guidance. ${ }^{1,4}$

The European Resuscitation Councilfurther recommends full PPE prior to starting chest compressions even if this results in a brief delay that may be associated with increased mortality and morbidity. They highlighted that the safety of staff is paramount. This comment should be interpreted in the light of a case series of 136 COVID-19 patients who required CPR, of whom only 18 ever had return of spontaneous circulation; four survived for at least 30 days and only one patient achieved a favourable neurological outcome at 30 days. ${ }^{10}$ This extremely poor prognosis is because most cardiac arrests in COVID-19 are pre-terminal events secondary to respiratory failure or multiorgan failure. ${ }^{3}$

In conclusion, the potential for recovery of the patient needs to be carefully balanced with the significant risk to the healthcare worker. In view of the intense anxiety of healthcare workers and in the absence of definitive evidence, we strongly recommend applying the precautionary principle and 


\section{"TThe Authors] strongly recommend applying the precautionary principle and support the use of full PPE before giving chest compressions during the pandemic.}

support the use of full PPE before giving chest compressions during the pandemic.

\section{Kamlesh Khunti,}

Professor of Primary Care Diabetes \& Vascular

Medicine, Diabetes Research Centre, University of

Leicester, Leicester, UK.

\section{Sebastian Straube,}

Professor and Division Director, Division of Preventive Medicine, Department of Medicine, University of Alberta, Edmonton, Alberta, Canada.

\section{Anil Adisesh,}

Associate Professor and Division Director, Occupational Medicine, Department of Medicine, University of Toronto and St Michael's Hospital,

Toronto, Canada.

\section{Xin Hui S Chan,}

Clinical Research Fellow, Centre for Tropical Medicine and Global Health, University of Oxford, Oxford, UK.

\section{Amitava Banerjee,}

Associate Professor in Clinical Data Science and
Honorary Consultant Cardiologist, Institute of Health Informatics, University College London, London, UK

\section{Trisha Greenhalgh,}

Professor of Primary Care Health Sciences, Nuffield Department of Primary Care Health Sciences, University of Oxford, Oxford, UK

\section{Provenance}

Commissioned; externally peer reviewed.

\section{Competing interests}

The authors have declared no competing interests

\section{Acknowledgements}

Kamlesh Khunti is supported by the National Institute for Health Research (NIHR) Applied Research Collaboration East Midlands (ARC EM) and the NIHR Leicester Biomedical Research Centre (BRC). The views expressed are those of the author(s) and not necessarily those of the NIHR, NHS or the Department of Health and Social Care.

DOI: https://doi.org/10.3399/bjgp20X711797

\section{ADDRESS FOR CORRESPONDENCE}

Kamlesh Khunti

University of Leicester, Diabetes Research Centre, Leicester LE5 4PW, UK.

\section{Email: kk22ale.ac.uk}

\section{REFERENCES}

1. World Health Organization. Modes of transmission of virus causing COVID 19: implications for IPC precaution recommendations. 2020. https://uww. who.int/news-room/commentaries/detail/ modes-of-transmission-of-virus-causingcovid-19-implications-for-ipc-precautionrecommendations laccessed 10 Jul 2020).

2. Majeed A, Molokhia M, Pankhania B, Asanati K. Protecting the health of doctors during the COVID-19 pandemic. Br J Gen Pract 2020; DOI: https://doi.org/10.3399/bjgp20X709925.

3. Fritz Z, Perkins GD. Cardiopulmonary resuscitation after hospital admission with covid-19. BMJ 2020; DOI: 10.1136/bmj.m1387.

4. Couper K, Taylor-Phillips S, Grove A, et al. COVID-19 in cardiac arrest and infection risk to rescuers: a systematic review. Resuscitation 2020; 151: 59-66.

5. Mahase E, Kmietowicz Z. Covid-19: doctors are told not to perform CPR on patients in cardiac arrest. BMJ 2020; DOI: 10.1136/bmi.m1282.

6. Public Health England. COVID-19: infection prevention and control guidance UK. 2020 https://assets.publishing.service.gov.uk/ government/uploads/system/uploads/ attachment data/file/886668/COVID-19 Infection_prevention_and_control_guidance_ complete.pdf (accessed 10 Jul 2020).

7. Center for Disease Control and Prevention. Interim infection prevention and control recommendations for patients with suspected or confirmed coronavirus disease 2019 (COVID19) in healthcare settings. 2020. https://uww. cdc.gov/coronavirus/2019-ncov/hcp/infectioncontrol-recommendations.html laccessed 10 Jul 2020).

8. European Resuscitation Council (ERC) European Resuscitation Council COVID-19 guidelines. 2020.

9. Resuscitation Council UK. Updated RCUK statement on PHE PPE guidance: 28 April 2020. 2020. https://www.resus.org.uk/ media/statements/resuscitation-council-ukstatements-on-covid-19-coronavirus-cpr-andresuscitation/statement-on-phe-ppe-guidance (accessed 10 Jul 2020)

10. Shao F, Xu S, MaX, et al. In-hospital cardiac arrest outcomes among patients with COVID19 pneumonia in Wuhan, China. Resuscitation 2020; 151: 18-23. 